\section{Practical issues in creating an evidence base for Library and Information Practice}

\section{Anne Brice, Andrew Booth}

\begin{abstract}
Authors
Anne Brice, Head of Knowledge \& Information Sciences, Public Health Resource Unit, Oxford, has research interests in evidence-based health care, knowledge quality and mentoring and professional development.
\end{abstract}

\begin{abstract}
Andrew Booth, Reader in Evidence Based Information Practice at ScHARR, University of Sheffield, has research interests in systematic reviews and Evidence Based Library \& Information Practice.
\end{abstract}

Email: anne.brice@dphpc.ox.ac.uk

\begin{abstract}
What are the practical issues in creating a knowledge base for the library and information profession? In this article the authors explore this topic from a practitioner-researcher perspective in order to identify the issues which arise from undertaking research, informed by experiences when conducting a qualitative systematic review.
\end{abstract}

The paper identifies barriers to using research, as perceived by library and information professionals, and discusses general issues encountered when undertaking research as a practitioner.

It reports the experiences of conducting a systematic review of qualitative research in the area of critical appraisal skills, a review spanning several disciplines. The authors select several factors of particular importance to illustrate the specific challenges of creating an evidence base in library and information practice. They highlight many examples of pitfalls and difficulties, but also the payback and rewards, of doing research.

\section{Introduction}

Evidence based practice carries an inherent assumption that all practitioners should be actively involved in applying evidence-based skills. The skills that you, as a practitioner, require range from those needed to undertake research, through asking of questions and identification of relevant research findings to application of research and its implementation in daily practice. In looking at barriers and issues that relate to conducting research, it is helpful to consider the broader relationship between practitioners and the research base. Indeed there may be a direct relationship between factors that influence how much we use research ourselves and how willing we are to undertake it.

\section{Barriers to using research}

In identifying barriers to using research within the library and information profession, it has been suggested that such problems stem from a communication and a cultural gap between researchers and practitioners (Genoni et al, 2004). Practitioners report constraints in time, language barriers, and problems with the physical availability of research outputs due to poor bibliographic control and indexing, compounded by lack of access to databases and full-text journals. As library and information practitioners we need to access not only library and information research, but also the research findings of education, marketing, business, computing and management, thereby adding to problems of access and availability (Winning, 2004). Poor practitioner use of research has also been attributed to the fact that research skills are not taught well, or not taught at all, in library education programmes, although the evidence on this is inconclusive (Genoni et al, 2004).

Even if we as practitioners are able to overcome such barriers as poor access and indexing we find that much of the library literature is of poor quality. Problems with reliability and validity undermine our confidence in its applicability and usefulness. Frequently we bemoan the fact that researchers do not appear to answer the questions to which we are most in need of 
answers (Farmer and Williams, 1999).

However, despite these barriers and problems with the literature once retrieved the available research remains underutilised (Genoni et al, 2004).

Crumley \& Koufogiannakis (2002) suggest that there is a pressing need to integrate publication and research into the workplace if development of the evidence base and application of research findings into practice is to be achieved. They argue that "It may even impel librarians to do better research and find other solutions if they are not satisfied with what they find in the literature".

Practitioner-researchers are become increasingly aware of the limitations of currently available research. A possible side effect of this is that some may become unwilling to engage in conducting research studies because of their concerns that constraints of time and funding necessarily lead to poor methodology or limited scope. Indeed a charge laid frequently against the research base for library and information practice, and its subsequent lack of research application, is that studies are too small to be reliable, too local to be transferable. The lack of adequate funding to support research projects may constrain how it is conducted, and research in librarianship tends to be conducted by individuals rather than teams.

Such challenges broadly fall into those concerning "consumption" of the evidence (addressed by developments in the use of ${ }^{*}$ critical appraisal checklists, journal clubs etc) and those related to "production" of the evidence base. It is on the latter that the remainder of this article will focus.

\section{Barriers to conducting research}

The hazards which face the small team or lone practitioner-researcher are well documented. Many of us report difficulties in identifying and acquiring funding for research projects. We also find it challenging to meet difficult-to-identify, or even hidden, costs such as the cost of obtaining studies through document delivery systems.
Time constraints include the time taken to plan the research and that spent applying for research funding. Practitioners need to be realistic about the time available to undertake the research and to balance this with day-to-day working pressures.

Understanding the processes for seeking ethical approval, and other governance issues involved in undertaking research presents a further hazard (Sen, 2003). In a recent example, a practitioner researcher in the health sector was required to apply for ethical approval in several organisations simultaneously, a process that took several months and caused delays in recruiting trial participants (Pearce-Smith, 2005). Indeed difficulties in recruiting appropriate participants, whether for observational or experimental studies, constitute a major problem for all librarian researchers.

Once the research is concluded, barriers to dissemination centre on publication. We may encounter difficulties in deciding in which type of publication to publish our research, i.e. library or sector specific. We may either be aware or unaware of factors related to publication bias. For example, some biases relate to extrinsic factors, i.e, belonging to the "wrong" institution, etc, while others relate to factors intrinsic to the research itself, i.e. positive publication bias relating to the unwillingness to publish research that shows either negative or small effects.

\section{Systematic reviews}

Many practitioners hold the misconception that an interest in research may only be realised by conducting primary research studies. We would encourage you to consider secondary research, so-called systematic reviews, particularly if you wish to apply research findings to practice (Beverley et al, 2003). A systematic review is:

.... a review of the evidence on a clearly formulated question that uses systematic and explicit methods to identify, select and critically appraise relevant primary research, and to extract and analyse data from the studies that are included in the review.

(NHS Centre for Reviews and Dissemination, Report 4 (2001)). 
The particular value of the systematic review method lies in bringing together several separately conducted studies, sometimes with conflicting findings, and synthesising their results. While this process shares with primary research the need to demonstrate use of explicit and rigorous methods it may be conducted from your own desk. Neither should you believe that such synthesis can only be applied to experimental studies such as randomised controlled trials. There is a growing body of expertise in the production of systematic reviews of qualitative studies and the documentation of methods associated with these. Indeed production of such reviews is fundamental to the success of other evidence based disciplines, notably in social care and education.

Conducting a systematic review, as with primary research, is a relatively complex endeavour. Typically it is carried out by small teams allowing sharing of skills and resources (Harris, 2005). While standards and guidelines are available to ensure that they are conducted consistently and objectively, reviews are vulnerable to several variables. First reviewers may differ in their motivation and prior experience. Then the complexity of the chosen review question may vary significantly. Next the characteristics of one discipline or subject area may prove more challenging than another discipline. As a consequence the features of studies that are identified for inclusion, including their rigour and their accessibility, may vary considerably. Finally environmental factors include the infrastructure(s) within which the review is being conducted, time and resource constraints, and political pressures from the commissioners of the review or other stakeholders. From these considerations we have selected several factors of particular importance to illustrate specific challenges in creating an evidence base in library and information practice.

Our systematic review aimed to characterise the principal barriers and enablers in the training and subsequent implementation of skills, knowledge and behaviour for "critical appraisal". Critical appraisal is "the process of assessing and interpreting evidence by systematically considering its validity, results and relevance to an individual's work" (Last, 1988). This systematic review was intended to operate at three levels, looking at health professionals in general; lessons specific to health librarians; and lessons that may be transferable to librarians working in other sectors. Our reasons for choosing this topic were drawn from a shared experience of teaching appraisal skills. We therefore shared a mutual interest in understanding whether teaching of these skills was both effective and useful. We were also keen to use the review to examine whether experiences found in different disciplines and sectors were transferable.

\section{Infrastructure Constraints}

The first constraint that we as a small team encountered related to the infrastructure within which we were conducting our research. This included such factors as access to databases, access to articles and the demands of both time and our "day jobs".

\section{Access to Databases}

Once a review team has formulated an answerable question, their first task is to develop a search strategy, which includes decisions on which databases to search. As has been noted by Law (2005)

...not all library managers have access to the breadth and depth of information required. There is limited access by practicing librarians to research literature, particularly those who are not employed by universities, or who are employed by universities that do not offer a library and information studies program. (Law, 2005)

The list of potentially relevant databases and sources can be extensive, given the breadth of disciplines that may be relevant to the question (Beverley, 2004). For our review an extensive list of sources were identified, including databases from the health care, education, business and social sciences disciplines, in 
addition to the library and information literature. Although we had access to resources through two separate university infrastructures not all the required sources were available. Of the total list of 22 databases or sources that had been identified as being potentially of interest, three major databases were inaccessible. You will therefore want to chart the availability and requirements for access to your key data sources before commencing your review.

\section{Access to Articles}

Having accessed and searched the relevant databases, inclusion/exclusion criteria are developed to help decide which studies are to be included in the review. However, the practitioner researcher may find it difficult to gain access to the full-text of articles, due to different access rights being available. As mentioned previously, costs for obtaining copies of papers through document delivery systems may be considerable, particularly for a broad review covering many disciplines. These problems are exacerbated by poor reporting which makes it difficult to apply inclusion and exclusion criteria without obtaining the full text.

\section{Time \& the Day Job!}

Law (2005) contends that most library practitioners do not have the time required to conduct a systematic review. Practically she estimates this time commitment as "approximately 1139 hours, or about 30 personweeks of fulltime work....depending on the number of citations." In addition, most systematic reviews require the time of a second reviewer, and as reviewers may be located in different organisations, expectations have to be made explicit from the outset.

Difficulties in balancing time between the review and other duties may vary depending on the nature of the post held by each reviewer. Whereas full-time researchers may experience pressure from the conflicting demands of other research projects, practitioner researchers encounter day-to-day pressures of their post, including political and organisational changes.
All these factors may impact on timetabling and availability making it impossible to schedule time in a realistic or planned manner.

\section{Requisite Skills and Competencies}

A second consideration relates to the skills and competencies required by the practitioner reviewer. Although these differ from those required by a primary researcher they are by no means less demanding. These include skills in searching databases, appraising the literature and in applying a growing body of systematic review methods.

\section{Searching the LIS and health databases}

Few librarians are skilled at retrieving literature that addresses questions in their own field (Beverley, 2004). In addition to the generic principles of literature searching, such as focusing your question, free text searching, thesaurus searching, using Boolean and proximity operators and limits, some specific search techniques, such as the development and use of methodological search filters, citation, author and hand searching need to be applied (Greenhalgh and Peacock, 2005).

A broad-ranging review may need to identify multiple study designs, for instance. This will have implications for searching, as very few LIS databases readily allow the searcher to limit results by either publication or study type. Similarly few LIS databases allow use of the more sophisticated methodological filters developed for searching medical databases (Jenkins, 2004).

Most LIS abstracts are unstructured and therefore very poor at describing study design and methodology (Booth, 1998). This makes the task of indexing the records doubly difficult for the indexer and, to compensate, the searcher is required to "second guess" terms that authors may have used. In addition problematic indexing requires the searcher to rely on freetext searching, despite known limitations of this approach. 
While training opportunities in literature searching are relatively plentiful these tend to focus on developing competencies amongst library users, rather than amongst the LIS profession itself, a situation that needs addressing. The practitioner researcher therefore needs to establish how competent they are in identifying the literature and to take steps to address any deficiencies through training (McGowan \& Sampson, 2005).

\section{Critical Appraisal}

Library and information professionals are increasingly required to demonstrate critical appraisal skills, in training their users and also as evidence based practitioners themselves. As discussed later, critical appraisal skills checklists have been developed specifically for use with the library literature, and can be used either by individuals in their own practice, by groups such as teams or in journal clubs. The assessment of both study design and quality also constitutes an essential part of the systematic review process itself.

\section{Systematic Review methods}

It takes much time and effort to acquire skills and knowledge of systematic review methods. This is particularly the case given that a preponderance of training opportunities falls upon conducting of quantitative systematic reviews, mainly in the health care field. As practitioner researchers we have individually acquired our skills in different ways - firstly through involvement in quantitative reviewing methods and also through an interest in how techniques of evidence based practice from one area (health care) potentially apply to the context of another (library and information practice). Opportunities exist for training in traditional systematic reviewing but an increasing interest in qualitative methods will multiply opportunities for other LIS practitioner researchers.

\section{Challenges from the process of review}

Constraints so far have focused on structural aspects. Additional constraints relate to the process of systematic review. These include difficulties in framing the question, identifying studies, assessing the literature, summarising the evidence and interpreting the findings.

\section{Framing the question}

Effectiveness reviews from within the health care field generally employ focused questions. In contrast, questions from the social science domain of library and information practice require a more holistic approach. Interest is in wider insights that relate not only to what is being done but also to how it is being done. Within health care questions are formulated or framed using an anatomy known as PICO (an acronym standing for Population, Intervention, Comparison and Outcome) cross-referenced to the particular study designs to be included. In a social science context attention has had to be broadened to include additional features such as the Setting and Perspective and to encompass wider Evaluation, not simply measurable Outcomes (Booth, 2004). In addition multiple forms of evidence may be required; some of these may not even constitute formal observational or experimental research and yet may make an important contribution to our understanding of a particular phenomenon.

\section{Identifying studies}

We have already highlighted the need for skills in retrieving literature. In addition to an overall proficiency with specialist technical features of the databases a practitioner researcher will also have to apply themselves to complementary methods of searching with an intensity not commonly encountered when they provide mediated searches to library customers. As Law concludes:

Although the growth in electronic resources has increased options for literature searching, it is still a process which requires a substantial level of skill, particularly in searching outside of the mainstream literature. As identified previously, many of the resources that are required to inform a management question (given its 
inherently interdisciplinary nature) are not consistently organized.

(Law, 2005)

Law goes on to identify that such skills need:

...constant practice and renewal in order to adapt to the changing information environment. The development and maintenance of searching skills requires a high level of motivation, which has been questioned by some writers.

(Law, 2005)

Information practice shares with such fields as education and management a poor quality of reporting. This makes it difficult to evaluate candidate studies based on bibliographic details, indexing and abstract alone (Law, 2005). In comparison health care literature has seen development of the "structured abstract", which summarises such components as objectives, design, setting, patients, main outcome measures, results and conclusion (Booth \& O'Rourke, 1997). Such a framework is thought to "considerably simplify the search and evaluation steps of a systematic review" (Law, 2005).

\section{Assessing the Literature}

A systematic review will typically involve quality assessment of candidate studies.

Numerous checklists have been devised for this purpose (Katrak et al, 2004). We have previously produced two such checklists, for User Studies and for Information Needs Analyses (Booth \& Brice, 2003; Booth \& Brice, 2004). However such checklists assume the use of a standard reporting format, such as Introduction Methods Results and Discussion (IMRAD). Unfortunately, studies in the information literature rarely pay attention to reporting of methodology. Likewise checklists include items that evaluate quality of research design, alongside those evaluating the quality of reporting. As a consequence it is more difficult to assess quality - a study rated poorly may not actually be a poorly-conducted study, it may simply be a poorly-reported one. Clearly the information literature needs to produce standards for reporting as developed in other fields (Golder et al, 2005).
A further ingredient of the process of synthesis, is the "hierarchy of evidence" (Evans, 2003) more accurately described as a "hierarchy of study designs". This places each study design in its relative position according to whether it is comparative or not, whether it is prospective or retrospective, and whether or not the population is allocated randomly to an intervention or . control group. This hierarchical approach has several limitations, particularly in areas where qualitative research and case studies are the norm. These are not less valuable forms of research, and perhaps require the hierarchy to be re-defined (Law, 2005).

We recognised at an early stage in our review that it was more important to trade-off the strength of a paper's findings against the weaknesses of its methodology rather than slavishly follow a hierarchy of evidence. Our thinking was stimulated by the concept of "signal versus noise" used as an alternative to hierarchies (Edwards et al, 1998). The "signal" represents the "strength of the message" from the research findings while the "noise" refers to any mediating factor that diffuses this message. For this review we attempted to identify three levels of "signal strengths". Signal level three (the weakest signal) is where an author makes an observation based purely on their own belief or experience. Signal level two is where such an observation or finding is ostensibly supported by data from research, a survey etcetera. Signal level one (the strongest level) is where a finding is directly supported by firsthand data on the phenomenon. We would encourage fellow practitioner researchers to consider approaching the evidence base in this way. Indeed we found such an approach wellsuited to a more holistic approach that acknowledges the complementary contributions of both research and non-research evidence.

\section{Summarising the Evidence}

The attraction of reviews or meta-syntheses for information science is self-evident. However controlled studies are not common within librarianship and while the question "does it work?" remains important, some would argue that equally important questions need to be 
answered around user acceptability and attitudes of staff. Library and information practice is a human-mediated discipline where we typically want to know "why something works?"

The implications of the above for our review were that we were able to use the emerging techniques of qualitative meta-synthesis to extract first-level themes or constructs from our identified studies. For example identified barriers may include "inadequate medical terminology knowledge", that librarians feel "uncomfortable with the new roles" and "lack of time [and clinical knowledge]". Once we had extracted and identified these themes we could then look for similarities across studies, what is known as "reciprocal translation". Once all themes have been grouped into similar categories we could look for the presence of "second order constructs". So, for our study we identified four categories of barriers; lack of confidence and efficacy, environmental factors, role limitations and technical knowledge. Finally we were able to explore the interrelationships between second-level constructs allowing us to construct a model to accurately describe the phenomenon. So, for example, we "discovered" that training in critical appraisal skills may help to counter barriers in technical knowledge. However it was also clear that once a librarian received a certain level of technical training they became more aware of exactly how complicated the subject area is. Rather than increasing their confidence and selfefficacy more training thus resulted in librarians becoming more aware of the limitations of their own knowledge and skills.

This progress from themes to categories to an overarching model is a very helpful and rewarding process but it does take much time and effort. It requires complete immersion in the data, often necessitating many iterations. In addition, "findings" from one investigator need to be challenged and validated by the other investigator to ensure that they are robust and are independently verifiable.

\section{Interpreting the Findings}

A major issue for evidence based practice in any discipline is applicability. To decide whether the results of a single study or a systematic review can be applied to your user population requires a subjective judgement on the similarity between that population and the population being studied. This is problematic even for the most robust of research studies. As a consequence appraisal checklists typically place the "burden of proof" with the reader.

In our review we advanced two conflicting hypotheses regarding applicability. The first is that health information workers are first and foremost health workers. In this case findings from the general health literature would apply equally to health librarians. Conversely, any model derived from our research would be applicable to other health professions. A contrary hypothesis is that health information workers are first and foremost information workers. In this alternative case, observations from health information workers have the potential to inform future experience with information workers from other sectors. In reality, we have little evidence to inform this debate about whether sector-specific factors (i.e. within health) are more important than profession-specific factors. However either standpoint will determine the overall applicability of our study.

Our dilemma is shared by at least one other systematic reviewer in a library context. Law (2005) systematically reviewed the literature on the impact of an MBA qualification on library management and practice. She comments:

Are there different cultural values in library organizations that might affect the acceptance of MBA studies? Does the high proportion of female workers in libraries create a different working culture? Does the placement of most libraries in the not-for-profit sector create a different environment for the application of skills learned as part of an MBA?

(Law, 2005)

She therefore concludes that:

...inferring application from the general management research literature to inform library 
management needs to be done with care, and suggests an area for future research.

(Law, 2005)

An even more problematic scenario would assume that sector and profession factors interact in a unique interplay placing health information workers in a category of their own. Such a conclusion would limit both the supply of evidence - we could then only include studies conducted with health information workers - and the applicability of the resultant evidence - it could then only be applied to that specific group. For our review we decided to investigate sector-specific and professionspecific factors separately. In effect, in the absence of a clear steer with regard to similarity of populations, we intend to conduct two separate reviews one within health and one within the more limited general library literature. We will then be able to look at results from both reviews and ultimately decide if their findings are indeed similar or whether there are nuances within each respective interpretation.

Perhaps the ultimate solution for the LIS evidence base is to turn attention away from the concept of applicability towards the concept of "relevance". In this case library practitionerresearchers would only claim that findings from the review are applicable to the context in which studies were conducted (in our case this is health information practice). Readers can then decide for themselves, provided that a review reports findings in specific enough detail, whether the findings resonate with their own context.

\section{Conclusion}

In conclusion then we have highlighted difficulties encountered when trying to conduct research as practitioner-researchers. We have proposed use of systematic review methodologies as a potential response to these. However such reviews carry their own challenges. These include structural issues relating to both Infrastructure and the need for Training. In addition there are process issues which relate to the generic stages of any systematic review and the specific requirements of our own evidence base. As well as endorsing a wider interest in the application of research synthesis within library and information practice we recommend the formation of partnerships and small teams of practitioner reviewers in order to harness the potential of shared skills and resources. We encourage a wider understanding within the profession of the holistic nature of "evidence" broadening beyond experimental quantitative studies to qualitative research, case studies and less substantial forms of reporting. Rather than seeking an elusive ideal threshold of study quality there is a need for us to mine the raw materials of the evidence base and to produce synthesized insights.

Rather than place the burden of applicability on the reviewers themselves, we would encourage our fellow practitioners, in their various library sectors, to assume the onus for relevance themselves. Our research skills should target the production and interpretation of the evidence base and should also be increasingly manifest - that is they should be both in evidence and in evidence!

\section{References}

Beverley, C (2004). Searching the library and information science literature. In: Booth, A. and Brice, A. (eds). Evidence Based Practice for Information Professionals:A Handbook. London: Facet Publishing, 89- 103.

Beverley, C.A., Booth, A., and Bath, P.A. (2003) The role of the information specialist in the systematic review process: a health information case study. Health Information and Libraries Journal 20(2),65-74.

Booth A. (1998). Testing the Lore of research, Library Association Record, 100(2), 654.

Booth, A (2004) Formulating answerable questions. In Booth, A and Brice, A (eds) Evidence Based Practice for Information Professionals: A handbook, London: Facet Publishing, 6I-70.

Booth, A. and Brice, A. (2003) Clear-cut?: facilitating health librarians to use information research in practice, Health Information and Libraries Journal 20 (Suppl I), 45-52. 
Booth, A and Brice, A. (2004) Appraising the evidence. In: Booth, A. and Brice, A (Eds) Evidence Based Practice for Information Professionals: A handbook. London: Facet Publishing, $101-118$.

Booth A. and O'Rourke, A.J. (1997) The value of structured abstracts in information retrieval from MEDLINE, Health Libraries Review 14(3), I57- 166.

Crumley, E, and Koufogiannakis, D. (2002) Developing evidence-based librarianship: practical steps for implementation, Health Information and Libraries Journal, 19(2), 61-70.

Edwards, A.G., Russell, I.T. and Stott, N.C. (1998) Signal versus noise in the evidence base for medicine: an alternative to hierarchies of evidence? Family Practice, I5, 319-22.

Evans, D. (2003) Hierarchy of evidence: a framework for ranking evidence evaluating healthcare interventions, Journal of Clinical Nursing 12, 77-84.

Farmer, J. and Williams, D. (1999). Are research priorities a priority for research? Health Libraries Review 16, 43-69.

Genoni, P., Haddow, G. and Ritchie, A (2004) Why don't librarians use research? In: Booth, A. and Brice, A (eds) Evidence Based Practice for Information Professionals: A handbook. London: Facet Publishing, 49-60.

Golder, S., Mather, L., Wright, K., and Glanville, J. (2005) What's on the web: recommendations for reporting research.J Health Serv Res Policy, I0(2), I 24- 125.

Greenhalgh, T. and Peacock, R (2005). Effectiveness and efficiency of search methods in systematic reviews of complex evidence: audit of primary sources. BMJ, 33 I (7524): 1064-5.

Harris, M.R. (2005) The librarian's roles in the systematic review process: a case study, J Med Libr Assoc. 93(I), 81-87

Jenkins, M. (2004) Evaluation of methodological search filters - a review, Health Information and Libraries Journal $21,148-63$.

Katrak, P, Bialocerkowski, A.E., Massy-Westropp, N., Kumar, S., and Grimmer KA (2004). A systematic review of the content of critical appraisal tools. BMC Med Res Methodol. 4,22.

Last JM (editor). A dictionary of epidemiology. New York: Oxford University Press; 1988.
Law, M (2005) The systematic review: a potential tool for research grounded library management URL: http://www.cais-acsi.ca/proceedings/2005/law_2005.pdf [18th February 2006]

McGowan, J. and Sampson, M. (2005) Systematic reviews need systematic searchers, J Med Libr Assoc, 93(I), 74-80.

NHS Centre for Reviews and Dissemination (200I). Undertaking Systematic Reviews of Research on Effectiveness: CRD Guidelines for those carrying out or commissioning reviews $2^{\text {nd }}$ edn (CRD Report No. 4), York: NHS CRD.

Pearce-Smith, N (2005). Randomised controlled trial comparing the effect of e-learning with a taught workshop on the knowledge \& skills of health professionals, IFMH Inform I6 (3), I7-I8

Sen, B.A. (2003) Research Governance: Implications for health information professionals. Health Information and Libraries Journal, 20 (1), 3-14.

Winning, A (2004). Identifying sources of evidence, In: Booth, A. and Brice, A. (eds). Evidence Based Practice for Information Professionals: A Handbook. London: Facet Publishing, 7I-88. 